\title{
Sexualidad y Adolescencia
}

\author{
II SIMPOSIO INTERNACIONAL DE PERINATOLOGIA
}

Dr. Germán Uriza Gutiérrez*

\section{INTRODUGCION}

En octubre de 1982 en la ciudad de Cúcuta, presenté una ponencia sobre Embarazo en Adolescentes en el Curso de Ginecología Infanto Juvenil que se realizó en dicha ciudad. Al revisar lo escrito en esa época, veo con preocupación que la situación en nuestro medio lejos de mejorar tiende a empeorarse. Los adolescentes en nuestro país, cada vez con más frecuencia tienen relaciones sexuales no protegidas, irresponsabilidad muy propia de su edad, que los lleva a enfrentar situaciones angustiosas como el embarazo indeseado, aborto provocado, madresolterismo, matrimonios y uniones forzosas, cesión de un hijo, abandono de la educación, hijos indeseados con su consecuencia el niño maltratado, prostitución y enfermedades sexualmente transmisibles, que los impulsa a tomar conductas evasivas catastróficas, como alcoholismo, drogadicción y suicidio.

Las situaciones antes planteadas, no pueden solucionarse con esfuerzos aislados de las entidades privadas; debe ser una acción conjunta de las entidades privadas y estatales, que reunan en un objetivo común a los adolescentes, padres, educadores, médicos, enfermeras, trabajadores sociales, psicólogos y sexólogos; con el fin de enfrentar estos hechos con decisión, sin timideces ni temores, sin pretender negar una realidad de la cual somos testigos muchas veces impotentes, todos los que tenemos que tratar en una u otra forma con los adolescentes que viven a diario verdaderas tragedias.

\section{PUBERTAD Ý ADOLESCENCIA}

Veamos las definiciones que el diccionario Larousse trae sobre estos dos hechos en la vida del ser humano; Pubertad del latín "Pubertas" es la época de la vida en que se manifiesta la aptitud para la reproducción y Adolescencia del latín "Adolescere, crecer", es el período de transición entre la infancia y la edad adulta (11). En medicina entendemos por pubertad, el período que transcurre entre la aparición de los primeros caracteres sexuales secundarios y la capacidad reproductora; en cambio con relación a la adolescencia, encontramos en la literatura muchas definiciones, algunas muy sencillas y otras muy extensas, siendo para mí la más completa la de la OMS, que dice: "La adolescencia es el período durante el cual el individuo progresa desde el punto de vista de la aparición inicial de los caracteres sexuales secundarios, hasta el de la madurez sexual; los procesos psicológicos del individuo y las formas de identificación evolucionan desde los de un niño, a los de un adulto; se hace la transición desde un estado de dependencia socioeconómica total a otro de relativa independencia (18). La pubertad entonces hace parte de la adolescencia, pero ésta se extiende mucho más allá de la madurez sexual; a lo largo del tiempo la disociación entre maduración biológica y maduración social es cada vez mayor, porque la pubertad está apareciendo cada vez más temprano. En Europa occidental la edad de la menarquía ha ido descendiendo en aproximadamente 10 meses por cada generación, según Park en 1845 , el $50 \%$ de las jóvenes menstruaba a los 15 años, en tanto que en 1962, el 50\% lo hacía a los 12 años (6). Según Tanner, esta disminución ha sido aproximadamente de 4 meses por cada decenio, y similar tendencia se encuentra en los Estados Unidos. Estos cambios se iniciaron a partir de la segunda mitad del siglo XIX y se tuvieron según Treolar y colaboradores, hasta la segunda mitad del siglo XX cuando se ha estabilizado en las tres últimas décadas a los 13 años (13).

Ginecólogo Obstetra. Profesor Asociado Universidad Javeriana. 
En Colombia la aparición de la menarquía se encuentra en promedio a los 12 años (9) y en consecuencia la primera ovulación a los 13 años, es decir, que una niña a los 13 años ya es capaz de reproducirse y sin embargo se encuentra muy lejos de ser adulta.

Según Papalia y Olds, "se es adulto cuando se han resuelto los problemas de la adolescencia: es descubrir la propia identidad, independizarse de los progenitores, desarrollar un sistema de valores propio con capacidad de formar relaciones amistosas y amorosas maduras e independientes" (19).

\section{SEXUALIDAD Y ADOLESCENCIA}

Las hormonas sexuales, estrógenos en la mujer y testosterona en el hombre, se empiezan a producir en forma creciente con la llegada de la pubertad, provocando la aparición de los caracteres sexuales secundarios en la mujer y en el hombre.

Simultáneamente con los cambios físicos que se producen en sus cuerpos, se comienza a manifestar el deseo sexual, con aparición en los muchachos de erecciones espontáneas como manipulación indirecta del pene que les causa sensaciones placenteras e inicialmente orgasmos secos, que con la maduración de los testículos termina con las poluciones nocturnas en los hombres y los sueños húmedos en las mujeres.

\section{A. MASTURBACION}

En la adolescencia es muy frecuente la masturbación, como medio de liberar la tensión sexual. Según Alfred Kinsey por lo menos 9 de cada 10 hombres y 6 de cada 10 mujeres se han masturbado regularmente en algún momento de su vida (26); para otros autores practican la masturbación casi el $100 \%$ de los hombres y entre el 40 y $70 \%$ de las mujeres (27). Existen opiniones en favor y en contra de la práctica masturbatoria. Sin embargo, considero que tiene grandes beneficios e incluso es realmente necesaria, permite al adolescente tener placer sexual sin el riesgo que la relación heterosexual a esta edad significa. Si lo hacen con prostitutas lo expone a enfermedades de transmisión sexual y le muestra ura respuesta sexual femenina falsa y perjudicial; si lo hace con su amiga, los expone a un embarazo indeseado con sus graves consecuencias.
Por otra parte permite el conocimiento de sus órganos sexuales, las zonas del cuerpo que producen al estimularlas placer sexual y las distintas fases de la respuesta sexual humana y por lo tanto sería de gran beneficio para su vida sexual futura en pareja.

Aunque ya la gente en su mayoría piensa que no es perversión pecaminosa, como creían nuestros abuelos, y aunque ahora los médicos reconocen que no tiene efectos físicos nocivos, todavía para algunas personas es difícil aceptarla como una fase natural y normal en el desarrollo sexual de una persona. Esta actitud tiene un fundamento histórico más que personal; en unos casos por la ortodoxia religiosa ya que la biblia considera la masturbación como "el abuso de sí mismo" considerando al "onanismo" como práctica abominable y pecaminosa. Sin embargo la opinión moderna es de que Onan al ser condenado por derramar su simiente en el suelo, no se estaba masturbando sino practicando el coitus interruptus (26). Por otra parte en el siglo XIX se produjo una manía antimasturbatoria creándose mitos sobre los efectos nocivos para la salud, ya que producía en los niños verrugas, impotencia, ceguera, y locura, mitos que se transmitieron de abuelos a padres y de éstos a sus hijos, muy difíciles de eliminar. En esta actitud social hacia la masturbación fueron decisivas las conferencias y escritos de Henry Varley quien en 1883 ante un auditorio de 3.000 hombres se refirió a ella como "El terrible y destructivo pecado del onanismo o abuso de sí mismo, práctica tan común como odiosa y dañina, que produce tendencia al resfriado, indigestión, depresión, somnolencia y ocio; práctica mortal y causa principal de enfermedad, decaimiento y muerte en nuestros jóvenes". (26).

Las teorías de Varley son ridículas, absurdas y causan risa en la actualidad, son fácilmente refutadas por hechos médicos, pero es difícil eliminar en la gente común, la idea de que la masturbación es algo malo y la vaga sospecha de que puede ser perjudicial. Muchos padres hoy reprenden enérgicamente a sus hijos si los sorprenden en prácticas masturbatorias, prohibiéndoles esta "anormalidad" y en esta forma inducen a sus hijos a relaciones heterosexuales, que si no están acompañadas de anticoncepción preventiva, sí son realmente peligrosas y exponen al adolescente a un embarazo indeseado. La masturbación en la adolescencia es una fase de transición fisiológica y en el adulto un impulso sexual sano cuando alguna razón impide el acto se- 
xual. Sólo se le puede considerar anormal cuando se prefiere la masturbación al coito, convirtiéndose en forma exclusiva de expresión sexual en una pareja en unión.

Por supuesto una obsesión constante en la masturbación indica alguna patología, como también la indica obsesionarse con cualquier otra actividad humana. Pero lo que hace falta es que se la apruebe totalmente como parte del proceso de maduración sexual y de la experiencia y que es no sólo normal y natural, sino necesaria y saludable. El disfrutar a solas de una comida, no quiere decir que no sea aun más agradable si se tiene una compañía querida con quien compartirla la mayor parte de las veces. La verdad del asunto parece ser que en muchas áreas el poder hacer cosas "uno sólo" significa que puede hacerlas todavía mejor en compañía de alguien (14).

\section{B. HOMOSEXUALISMO}

En los primeros años de la adolescencia las actividades escolares y los juegos, son generalmente con individuos del mismo sexo y como se están produciendo cambios físicos en sus órganos sexuales y se empieza a experimentar deseo sexual, es muy frecuente que grupos de muchachos y muchachas del mismo sexo se muestren los órganos genitales e incluso entre los hombres pueden llegar a masturbaciones en grupo mientras miran fotos o películas pornográficas. Puede presentarse incluso verdadera atracción sexual por un amigo o amiga del mismo sexo, situación transitoria que desaparece con la maduración y es lo que se conoce como la homosexualidad transitoria de la adolescencia (20) que desaparece cuando se establecen las relaciones heterosexuales. Me acuerdo en este momento de una paciente de mi consultorio que solicitó una cita urgente. Al entrar esta mujer no podía hablar por el llanto, pero el motivo de su consulta no era ginecológico ni obstétrico, sino que su hijo de 8 años era homosexual. $\mathrm{Al}$ interrogarla sobre el por qué asumía que su hijo era homosexual, me contó que lo había encontrado explorándose los genitales con un hermano y que por esta razón los había llevado a la psicóloga quien le había confirmado el diagnóstico de homosexualismo. Evidentemente la psicóloga estaba totalmente equivocada, ningún niño a esta edad puede ser homosexual y posiblemente influida hasta la exageración por escuelas que consideran la homosexualidad como una enfermedad psiquiátrica, y llevó a esta madre a una situación de terror y angustia ante un hecho frecuente y normal.

En mucha de la literatura psiquiátrica se encuentra la suposición de que la homosexualidad es un problema patológico y que la mayor contribución que la psiquiatría puede aportar al problema es buscar cómo curarlo. Sin embargo, muchos psiquiatras piensan que excluyendo la desadaptación sexual, es perfectamente posible que un homosexual sea feliz y que logre un buen ajuste social (14).

Los estudios psicoanalíticos que afirman que son las madres neuróticas las que convierten a sus hijos en homosexuales, o la investigación del Dr. Irving Bieber titulada "Homosexualidad, un estudio psicoanalítico", que concluye que "un padre constructivo que dé apoyo y que sea cariñoso con sus hijos quita la posibilidad de un hijo homosexual" adjudican la responsabilidad de todo homosexual a sus padres. Esta situación es la que ha llevado a la afirmación de un educador de vida familiar, quien dice: "Los padres pueden soportar casi todo, matrimonios entre razas diferentes, promiscuidad, embarazos fuera del matrimonio si fuera necesario, pero puedo asegurar que hay algo que nunca verá usted en su vida y es que los padres acepten verdaderamente las relaciones homosexuales de sus hijos; van en contra de siglos de condicionamiento" (14).

\section{RELACIONES HETEROSEXUALES}

Cuando los adolescentes inician relaciones heterosexuales, lo hacen influidos por diversos factores de orden social, familiar o individual. En nuestra sociedad se observa una clara diferencia en cuanto a las conductas sexuales permitidas si es hombre o es mujer. Algunos padres llegan incluso a estimular a su hijo varón para que tenga relaciones sexuales con prostitutas con el fin de protegerlo contra la masturbación o el homosexualismo, exponiéndolo a riesgos graves de enfermedades sexualmente transmisibles. En cambio a la mujer se le exige un estado de castidad prenupcial y una excesiva valoración de la virginidad (22). En lugar de establecer un culto al hímen mitológico diciendo a las niñas que si no se mantienen castas "perderán algo verdaderamente bello y muy especial". Se les debe informar de los riesgos del embarazo indeseado, las enfermedades sexualmente transmisibles y la desorganización social que para ellas significa el madresolterismo, el matrimonio forzoso, la cesión de un hi- 
jo y el aborto. Conflictos familiares, violencia intrafamiliar, incomunicación, desafecto y autoritarismo; son condiciones que generan en el adolescente una necesidad desmedida e incontrolable de afecto que busca canalizar neuróticamente en la relación de pareja, en donde la gratificación sexual no apunta siempre a la satisfacción de un deseo, sino a una entrega sumisa, incondicional, sin ninguna defensa del ego (22). Algunos investigadores encuentran también que la sobreprotección de los hijos únicos dificulta el aprendizaje de relaciones de competencia y la hace vulnerable ante figuras de liderazgo no parental y en otras agudiza la necesidad de independencia y autoafirmación encontrando en la relación sexual la validación de su papel de adulta.

El sexo es una de las pocas maneras mediante las cuales una adolescente puede rebelarse con eficacia contra la sociedad, los padres o sus compañeros. Ella puede hacerlo conscientemente cuando responde a una afrenta personal o subconcientemente en una rebelación contra la sociedad que la amenaza o le niega sus derechos individuales o la oportunidad de liberarse de las restricciones sociales (29). La actividad heterosexual en la adolescente, sea voluntaria o involuntaria, siendo casada, en unión libre, por incesto, violación o prostitución la expone a un embarazo en una edad que significa un alto riesgo.

\section{EMBARAZO EN ADOLESCENTES}

La pubertad precoz y su madurez temprana, cuando se establece una relación heterosexual, entre los adolescentes sin la adecuada utilización de las técnicas anticonceptivas, expone a la niña adolescente a un embarazo en su gran mayoría indeseado a una edad en la cual no está preparada ni fisiológicamente ni psicológicamente para encarar la responsabilidad que la maternidad exige. Es decir, que el embarazo en la adolescencia expone a la niña a un riesgo mayor de sufrir un daño. Para entender mejor el concepto de enfoque de riesgo vemos las siguientes definiciones (3):

1. RIESGO: Se define como la posibilidad mayor o menor de tener un resultado o daño.

2. FACTOR DE RIESGO: Es toda condición o característica posible de medir en un indivi- duo o comunidad, que se asocia con una probabilidad aumentada de presentar un daño.

3. La posibilidad se asume entre el valor cero (0), cuando no existe la más mínima posibilidad de que se produzca el daño y uno (1), cuando la posibilidad de que ocurra es del $100 \%$.

El embarazo en la adolescencia tiene implicaciones sociales e implicaciones médicas.

\section{A. IMPLICACIONES SOCIALES}

1. La adolescente y su hijo representan un agudo problema dentro de su familia y la comunidad. La mayoría de las veces su aporte económico es nulo y constituye una nueva carga al presupuesto familiar (28).

2. El embarazo en adolescentes presenta la característica particular de que en la mayoría de los casos se producen sin mediar vínculos matrimoniales (22) y la madre soltera y su hijo siguen privados en muchos países, de muchos derechos legales y beneficios sociales (29).

3. Su experiencia como madre, y la responsabilidad que la maternidad exige, le demanda un largo período de adaptación para con el nuevo ser y consigo misma y en ocasiones crea trastornos psicológicos (29). Es muy frecuente la depresión que las induce al alcoholismo y la utilización de drogas (10).

4. El rechazo que muchas de nuestras comunidades imponen a la madre soltera, es solucionado frecuentemente con un matrimonio precoz abocado al fracaso $(4,15,29)$.

5. El abandono de la escuela se asocia a la maternidad temprana con niveles educativos bajos. Muy pocos sistemas de enseñanza tienen previsto el acomodo de las muchachas embarazadas con sus hijos en las actividades normales de la escuela, de hecho la política vigente en algunos países constituye en negarles la entrada, por temor de que puedan influir desfavorablemente en otros alumnos $(22,29)$.

6. En el aspecto económico, la madre adolescente soltera está obligada a aceptar trabajos de baja remuneración y mínimo status; con jor- 
nadas excesivas y formas inestables e irregulares de contratación (22)

7. La cesión del hijo es muy frecuente en la madre adolescente, en algunos contextos sociales en que existen familias extensas y muy numerosas, la asistencia de los niños fuera del matrimonio pueden asumirla los padres o parientes de la madre soltera evitando así la necesidad de la adopción legal (29). Cuando el recurso de la adopción legal es posible, ésta debe ser una solución que sólo incumbe a la madre adolescente. Un punto difícil respecto a la madre que entrega su hijo en adopción, es si debe ver al bebé, alimentarlo y darle un nombre antes de cederlo. Muchos autores defienden esta teoría (29).

8. El aborto provocado es la otra alternativa de solución que tiene la adolescente embarazada cuando la legislación de su país lo permite. En Colombia todo aborto, no importando la causa, es ilegal y realizado en condiciones no médicas pone en grave riesgo la vida de la mujer que se somete a él. El aborto séptico en Colombia es la primera causa de mortalidad materna (28).

9. Es frecuente la impreparación del personal de salud para hacer frente a los problemas psicosociales que plantean las adolescentes embarazadas. Los sistemas de envíos a clínicas especializadas rara vez establecen diferencias entre la mujer adulta y la adolescente, pues se parte del supuesto de que las necesidades son las mismas y que llegan al servicio asistencial con esperanzas y experiencias similares (29).

10. En su mayoría el embarazo en la adolescencia como lo afirma la Federación de la paternidad planeada, es indeseado (12) y si no recurre al aborto, adopción o su nueva situación es aceptada por la adolescente, la somete a tener al hijo no deseado con sus graves consecuencias para el recién nacido como son: El infanticidio, homicidio inconsciente, niño golpeado, abandono, rechazo y aceptación tardía (27).

11. Finalmente, es posible que una adolescente con un embarazo indeseado, en un ambiente social y familiar hostil, que no encuentre apoyo ni alternativa de solución oportunos, recu- rra al alcohol, drogas o tome la decisión extrema del suicidio.

\section{B. IMPLICACIONES MEDICAS}

1. Atención prenatal: A la impreparación del personal de salud, en la atención del adolescente, mencionada anteriormente, se suma la asistencia tardía, irregular o nula al control prenatal como resultado de su negativa, miedo y vergüenza que la adolescente experimenta por su embarazo.

2. La Toxemia, anemia, alteraciones psicológicas y psiquiátricas, partos pretérmino, niños de bajo peso, distocias, anomalías congénitas del recién nacido y mayor tasa de mortalidad perinatal e infantil, son algunos de los problemas obstétricos-perinatales que se presentan con más frecuencia en las madres adolescentes que en los grupos de mayor edad (2, 7, 9, 23, 29).

\section{PREVENCION}

Siendo el embarazo en la adolescencia un alto riesgo, es preciso que durante este período evolutivo no se produzca un embarazo. Para lograr este objetivo debemos brindar:

1. EDUCACION: Educar sin importar si la joven es sexualmente activa o no, sobre sus órganos reproductivos, fecundación, sexualidad y anticoncepción (21). Hacer énfasis sobre los peligros médicos y sociales que conlleva al embarazo en esta edad y que ningún método anticonceptivo brinda el $100 \%$ de protección, por lo tanto sería mucho más conveniente posponer las relaciones heterosexuales para cuando sea una mujer adulta (29).

2. ANTICONCEPCION: Si la adolescente está casada, en unión o es soltera pero sexualmente activa, debe brindársele anticoncepción preventiva eficaz, como única forma de luchar contra el embarazo indeseado y sus consecuencias, el aborto, el hijo indeseado y el suicidio (29).

\section{EDUCACION SEXUAL}

Insistir sobre la necesidad y la importancia de la educación sexual parecería para algunos innece- 
sario, pero si nos detenemos a analizar los conceptos que sobre el tema fueron emitidos por eminentes Psiquiatras colombianos en dos artículos publicados por El Espectador, uno "El niño y el sexo" (7) y el otro "Al rescate del recato" (25), comprenderán ustedes mi preocupación y la de muchos otros colegas, ante el peligro de regresar a épocas que todos creíamos hace mucho tiempo superadas en Colombia.

En la declaración titulada "El niño y el sexo" del Comité de Psiquiatría del niño y el adolescente de la Sociedad Colombiana de Psiquiatría, suponen que en Colombia se está promoviendo y generalizando la Paidofilia, con la cual ninguno de nosotros está de acuerdo. Pero los comentarios hechos por el Dr. Francisco Cobos en el artículo "Al rescate del recato" sobre la anterior declaración en los que responsabiliza a la educación sexual, de las relaciones sexuales de los niños con los adultos, es totalmente absurdo. Veamos algunas de estas afirmaciones del Dr. Cobos: 1. "La mayoría de las madres de la clase media salen realmente desnudas y con muy pocas prendas de vestir delante de los niños, sin saber, o sin tener idea que estos niños se excitan y que entran en un conflicto enorme". 2. "La educación se. xual temprana es un error gravísimo". 3. "Los conocimientos sexuales los debe adquirir el niño del grupo de copartícipes en el juego así sean equivocados". 4. "Es más importante para el niño los conocimientos equivocados sobre el sexo ya que el mito es muy importante". 5. "Las familias no se deben bañar juntas". 6. "Se debe abolir la costumbre de ver televisión los niños y los padres en el lecho conyugal". 7. Ante las anteriores afirmaciones el Dr. Juan Mendoza Vega dice: "Pretender que los niños reciban sus primeras nociones sobre el sexo del grupo de copartícipes en el juego, vale decir, de sus compañeritos de clase o de barrio y así sean equivocados esos conocimientos iniciales, resulta de tal modo absurdo que apenas podría provocar una sonrisa de conmiscración"; "la presunta prohibición de reunirse alrededor de los padres en el gran lecho conyugal, para ver la televisión o simplemente para conversar un rato juntos es igualmente exagerada hasta lo ridículo" (16).

Los Doctores Alvaro Villar Gaviria de la Sociedad Colombiana de Psiquiatría y el Dr. German Ortiz Umaña de la Sociedad Colombiana de Sexología, tampoco están de acuerdo con las afirmaciones del Dr. Cobos y al respecto hacen las siguientes recomendaciones: " 1 . No atribuyamos a los niños los problemas y las ansiedades, producto de nuestra mala educación sexual. 2. No los avergoncemos de sus propios cuerpos exhibiendo la vergüenza que sentimos por los nuestros. 3. No les mintamos, no los engañemos, porque la mentira y el engaño son formas de hacer violencia. 4. Acariciemos a nuestros hijos y expresémosles nuestro afecto, con el convencimiento de que las manifestaciones físicas de cariño y amor nunca tienen el signific ado morboso que distorsionadamente algunos le dan". (1). La Sociedad Colombiana de Sexología con la firma de la Psicóloga María Ladi Londoño y los Doctores German Ortiz Umaña y Helí Alzate entregaron una declaración a la opinión pública donde expresan su desacuerdo con el Dr. Cobos. Sobre esta declaración, el Dr. Juan Mendoza Vega dice: "Para los sexólogos como para cualquier persona de buen juicio, la educación sexual no sólo debe mantenerse sino perfeccionarse, por ser componente indispensable del desarrollo normal humano" (17). La sexualidad es un comportamiento humano y por lo tanto no existe alternativa entre conocerla y no conocerla, es decir que la educación sexual debe iniciarse tan pronto el niño y la niña son conscientes de su propio sexo, su enseñanza y orientación deben empezar en la etapa pre-escolar, en el hogar y son los padres quienes serán los primeros cducadores". La educación scxual no es una alternativa de los padres frente a los hijos, o de los maestros frente a sus alumnos, no existe la posibilidad de escoger entre dar educación sexual o no darla; es obligatorio y es un derecho que todo ser humano tenga iguales oportunidades de conocer y gozar de su sexualidad (30).

\section{CONCLUSIONES}

Siendo la adolescencia un período de transición entre un niño y un adulto, caracterizada por rápidos cambios físicos y psíquicos que se traducen en un estado de inestabilidad emocional, no podemos convertirnos los adultos en un factor más de desestabilización. Por lo tanto es nuestra obligación ayudar al adolescente a lograr un desarrollo armónico de su personalidad obteniendo así una adecuada maduración.

Debemos educar sin importar que el adolescente sea sexualmente activo o no, sobre sus órganos reproductores, ciclo menstrual, fecundación, 
sexualidad humana y anticoncepción. Es necesario evitar que durante este período ocurra un embarazo indeseado, por lo tanto la educación no debe limitarse al nivel de la información, es indispensable hacer difusión dentro de los adolescentes sobre las entidades dedicadas a brindar atención integral al adolescente, las cuales deben tener por ley, autorización para dar información y suministro de anticonceptivos a los que lo soliciten o requieran. $\mathrm{Mu}-$ chas investigaciones han demostrado que cuando las adolescentes solicitan un método anticonceptivo, el $70 \%$ ya están embarazadas y en su gran mayoría han transcurrido 12 meses de relaciones heterosexuales no protegidas. Esto demuestra la afirmación falsa de que la educación sexual y el conocimiento o suministro de anticonceptivos, son los

\section{BIBLIOGRAFIA}

1. ALVARADO MARIA CRISTINA: "No al terror por el sexo", El Espectador, jueves, diciembre 17 de 1981.

2. BALLARD W. GOLD EL: "Aspectos médicos y de salud de la reproducción en la adolescencia”, Clínicas Obstétricas y Ginecológicas, junio 1971: 338-66.

3. BARRAGAN JAIRO: "Instrumentos de predicción", Revista Colombiana de Obstetricia y Ginecología. Vol. XXXVIII No. 5. Oct. 1987.

4. BILLY JO; LANDALE NS; MCLAUGHLIN SD.: "The effect of marital status at first birth on marital dissolution among adolescent mothers", Demography 1986, Aug; 23 (3) 329-49.

5. BOLTON G.: "Uso de anticonceptivos para adolescentes". Clínicas Obstétricas y Ginecológicas. Vol. III 1981: 985: 95.

6. CABRERA RENE: "La reproducción en la adolescencia" APROFA. Chile.

7. COMITE DE PSIQUIATRIA DEL NIÑO Y EL ADOLESCENTE. SOCIEDAD COLOMBIANA DE PSIQUiATRIA. “El Niño y el Sexo”. El Espectador, Lunes, Dic. 7, 1981.

8. CHIO ILEANA; ESPINOSA ANGEL; POUYMINO TARARA; PEREZ RODOLFO; ALVAREZ ISABEL: ECHAVARRIA ANGEL: "Mortalidad perinatal en embarazadas adolescentes". Rev. Cubana de Obstetricia y Ginecología Vol. 13. No. 1, enero-marzo, 1987. que inducen al adolescente a una actividad sexual premarital o promiscua.

Por otra parte pensar que la adolescente que ha experimentado un embarazo indeseado ya aprendió la lección, es una utopía, por lo general la niña que ha iniciado su vida sexual heterosexual, la va a continuar, por lo tanto es necesaria la anticoncepción preventiva en toda adolescente en el post parto o en el post-aborto.

Ayudemos a nuestros hijos a pasar por el difícil período de la adolescencia sin traumas que obstaculicen su normal desarrollo psíquico y físico para que al final de la misma se conviertan en adultos productivos y útiles a la sociedad.

9. DUARTE CONTRERAS ALBERTO: “ ¿El embarazo en adolescentes es siempre de alto riesgo?”. Rev. Col. de Obstetricia y Ginec. Vol. XXVI Nov.-Dic. 1975; 385-95.

10. ELSTER ARTHUR; PANZARINE SUSAN; McANARNEY ELIZABETH: "Causes of adolescent pregnancy", Medical aspects of human sexuality vol. 14 No. 7, july 1980.

11. GARCIA PELAYO RAMON: "Pequeño Larousse Ilustrado". Ediciones larousse, 1981.

12. GODENNE G. "El embarazo en las adolescentes solteras", Aspectos psicológicos en Ginecología y Obstetricia II; Ediciones PLM, México 1981.

13. GRUPO DE TRABAJO SOBRE NUTRICION Y EMBARAZO EN LA ADOLESCENCIA, ACADEMIA NACIONAL DE CIENCIAS DE WASHINGTON D.C.: "Relación entre la nutrición y el embarazo en la adolescencia", Clínicas Obstétricas Ginecológicas, Junio 1971; 367:92.

14. LE SHAN EDA: "Los adolescentes y la revolución sexual". Editorial Pax México, 1975.

15. Mc LAUGHLIN SD; GRADY WR; BILLY JO; LANDELE NS: WINGES LD.: "The effects of the sequencing of marriage and first during adolescense". Fam. Plann perspect 1986 Jan-Feb.; 18 (1): 12-8.

16. MENDOZA VEGA JUAN: "Retorno de los Brujos", El Espectador, Jueves, Dic. 17, 1981. 
17. MENDOZA VEGA JUAN: "Dicen los que saben". El Espectador enero 18, 1982.

18. OMS. "El embarazo y el aborto en la adolescencia". Sec. Inf. Tecn. No. 583, 1975.

19. PAPALIA D; OLDS S.W.: "A child's world, infancy, Thorugh adolescense”. New York: Mc Graw-Hill, 1975.

20. PELAEZ PAULA: "Desarrollo integral del adolescente. Características biológicas, psicológicas y sociales de la adolescencia". Sexualidad humana y relaciones personales. Segunda edición: 117-30. IPPH/ RHO. Nueva York, 1983.

21. PRIETO DE SOSA ROSA INES: "Embarazo de la adolescente". Sexualidad humana y relaciones personales. Segunda edición: 323-31 IPPH/ RHO. Nueva York, 1983.

22. RICO DE ALONSO ANA: "Madres solteras adolescentes". Plaza y Janés. 1a. Edición, feb. de 1986.

23. RYAN GEORGE JR; SCHNEIDER JACK: “Complicaciones Obstétricas en los adolescentes". Clínicas Obstétricas y Ginecológicas, Vol. 4, 1978.

24. SANCHEZ TORRES FERNANDO: "Adolescencia y embarazo" El Tiempo.
25. SANTOS CECILIA: "Al rescate del recato". El Espectador, lunes dic. 7, 1981.

26. TONER BARBARA: "Encyclopedia of Loue and sex". Dismail, S.A. Alcalá: 96, Madrid 7.

27. TRIAS MIGUEL: "El hijo indeseado". Editorial Retina Sep. 1974.

28. URIZA GERMAN; LOPEZ GUILLERMO; RIAÑO G.: "Estudio Hospitalario de Mortalidad Materna”. Rev. Col. de Obst. y Gin. Vol. XXXIII. No. 5 Sept.-Oct. 1982.

29. URIZA GERMAN: "Embarazo en adolescentes". Rev. Col. de Obst. y Ginec. Vol. XXXIV No. 2, 1983.

30. URIZA GERMAN: "Importancia de la educación sexual en un programa de reproducción humana". Monografías de la Corporación Centro Regional de Población. Febrero de 1986: Vol. 20: 108-14.

31. ZUCKERMAN BS ; AMARO H; BEARDSLEE W.: "Mental Health of adolescent mothers. The implication of depression and drug use". J. Dev. Behau Pediatr.1987. Apr. 8 (2): 111-6.

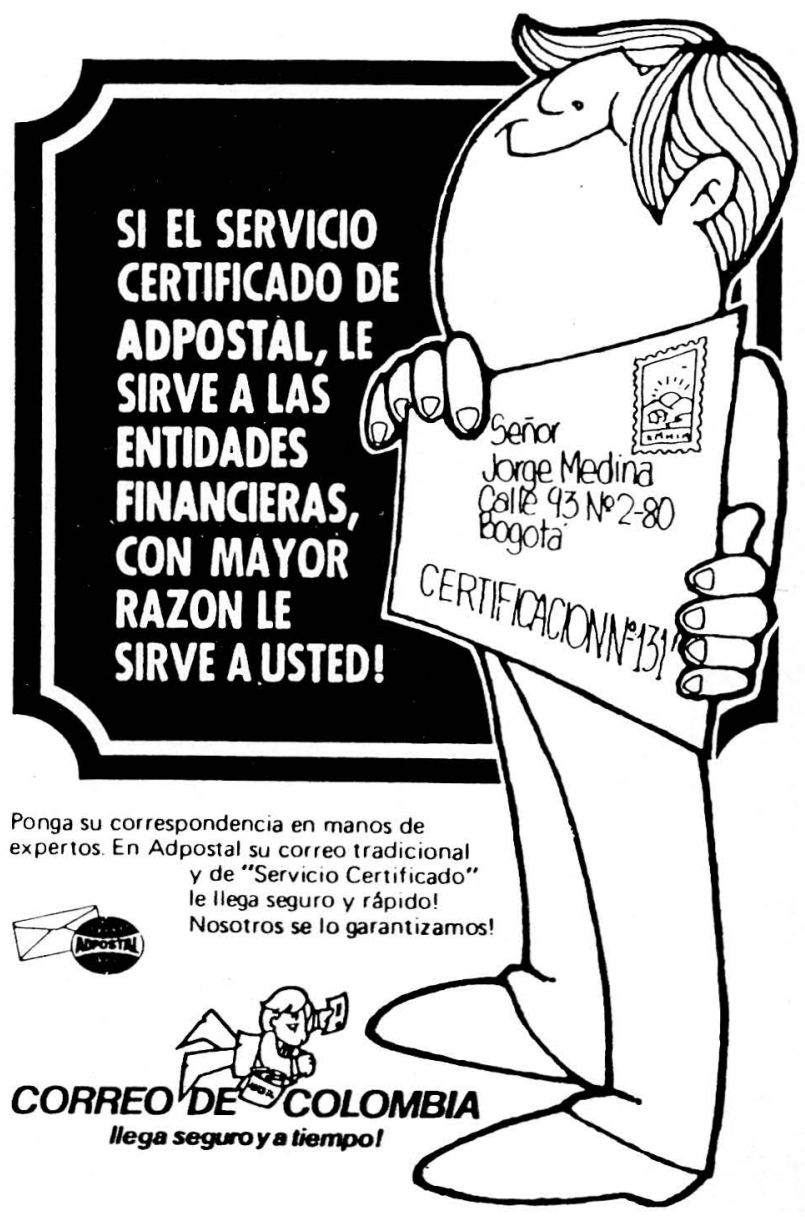

